\title{
Role for Target of Rapamycin (mTOR) Signal Pathway in Regulating Neuronal Injury after Intracerebral Hemorrhage
}

\author{
Jie-Ping Wang ${ }^{a}$ Meng-Yu Zhang ${ }^{b}$ \\ aDepartment of Rehabilitation, ${ }^{b}$ Department of Hepatobiliary Surgery, Southwest Medical University \\ Affiliated Hospital, Luzhou City, China
}

\section{Key Words}

Intracerebral hemorrhage $\cdot \mathrm{mTOR} \cdot$ Rapamycin $・$ Cytokines $・$ Caspase-3

\begin{abstract}
Background/Aims: Mammalian target of rapamycin (mTOR) is a serine/threonine protein kinase and activation of its signal pathway plays an important role in regulating protein growth and synthesis as well as cell proliferation and survival. In the present study, we examined the contribution of mTOR signal and its downstream products to brain injuries induced by intracerebral hemorrhage (ICH) in rats. Methods: Western Blot analysis was employed to examine the protein expression of mTOR and its downstream pathway and Caspase-3. ELISA was used to measure pro-inflammatory cytokines (PICs) and vascular endothelial growth factor (VEGF). Additionally, neurological Severity Score and brain water content were used to indicate neurological function and brain edema. Results: The protein expression of $\mathrm{p}-\mathrm{mTOR}$, mTOR-mediated phosphorylation of 4E-binding protein 4 (4E-BP1), p70 ribosomal S6 protein kinase 1 (S6K1) pathways were amplified in ICH rats compared with controls. Blocking mTOR using rapamycin significantly attenuated upregulation of PICs, namely IL-1 $\beta$, IL- 6 and TNF- $\alpha$, and Caspase- 3 indicating cell apoptosis, and promoted the levels of VEGF and its subtype receptor VEGFR-2 in brain tissues. Moreover, the effects of rapamycin were linked to improvement of neurological deficits and increased brain water content observed in ICH rats. Conclusion: Activation mTOR signal is engaged in pathophysiological process during ICH and blocking mTOR pathway plays a beneficial role in regulating neuronal tissues via PIC, apoptotic Caspase-3 and VEGF mechanisms. This has pharmacological implications to target specific mTOR and its downstream signal pathway for neuronal dysfunction and vulnerability related to $\mathrm{ICH}$.
\end{abstract}

\section{Introduction}

In general, intracerebral hemorrhage (ICH) is caused by brain trauma and also considered as the second most common cause of stroke because it occurs spontaneously in 


\section{Cellular Physiology Cell Physiol Biochem 2017;41:145-153

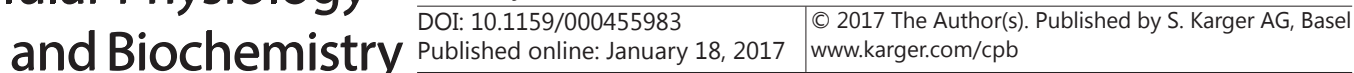 \\ Wang et al.: Activation of mTOR by Intracerebral Hemorrhage}

hemorrhagic stroke. Non-traumatic ICH is observed to lead to a spontaneous bleeding into the brain tissue and cause a high morbidity and mortality [1]. A number of pathophysiological changes are observed following ICH including brain edema, blood clotting and neurological deficits $[2,3]$. There are many risk factors to be related to non-traumatic ICH. Hypertension and hyperglycemia the common and important causes of ICH [4]. It has been reported that patients with a higher blood pressure and diabetes have a greater risk to suffer ICH and have the worse outcomes after ICH than patients without diabetes [5-7]. Animal studies further suggest that multiple pathways such as inflammation and oxidative stress are involved in hypertension and diabetes and they also deteriorate neurological deficits after ICH $[8,9]$. Nevertheless, the mechanisms responsible for ICH remain to be determined.

Mammalian target of rapamycin (mTOR) including complex 1 (mTORC1) and complex 2 (mTORC2) is a serine threonine protein kinase. Specifically, mTORC1 is more sensitive to rapamycin and its activation promotes the phosphorylation of downstream effectors such as p70 ribosomal S6 protein kinase (p70S6K), which further governs mRNA translation [10]. The mTORC1 is well known for its critical roles in the regulation of protein synthesis and growth, but the precise signals for dysregulation of mTOR in the process of ICH are largely lacking. Accordingly, in the present study, we examined responsiveness of mTOR signal pathway in the brain tissues of control rats and ICH rats, and further determined if proinflammatory cytokines (PICs, namely IL-1 $\beta$, IL- 6 and TNF- $\alpha$ ) and Caspase- 3 as an indicator of neuronal apoptosis were engaged in the effects of mTOR signal on neurological deficits evoked by ICH.

Vascular endothelial growth factor (VEGF) is an important signaling protein engaged in both vasculogenesis and angiogenesis, which lead to restoring the blood supply to tissues [11]. It has been shown that VEGF stimulates endothelial cell mitogenesis and cell migration, but also enhances microvascular permeability [11]. Moreover, it has been reported that VEGF is up-regulated after the ICH and this likely improves the permeability of blood-brain barrier, reduces brain edema formation and promotes the recovery of brain injuries $[12,13]$.

It should be noted that VEGF regulates vascular development, angiogenesis and lymphangiogenesis by binding to a number of VEGFRs [14, 15]. There are three main subtypes of VEGFR, numbered 1, 2 and 3. The function of VEGFR-1 is less defined, although it is required for the recruitment of haematopoietic stem cells and the migration of monocytes and macrophages $[14,15]$. VEGFR-2 appears to mediate almost all of the known cellular responses to VEGF and it is critical for vascular endothelial cell development and regulates vascular endothelial function [14]. VEGFR-3 regulates lymphatic endothelial cell function and mediates lymphangiogenesis in response to VEGF $[14,15]$. Considerable evidence shows that VEGFR-2 is specific intracellular signal cascades leading to proliferation, migration, survival and increased permeability, each of which contributes to the angiogenic response [14]. Accordingly, in the present study, we examined the involvement of VEGF and its subtype receptor VEGFR-2 in mTOR signal pathway in the process of neurological deficits after ICH.

We hypothesized that ICH increases the protein expression of p-mTOR, mTORmediated phosphorylation of 4E-binding protein 4 (p-4E-BP1), p70 ribosomal S6 protein kinase 1 (p-S6K1) pathways. We further hypothesized that a blockade of mTOR attenuates upregulation of PICs and Caspase- 3 induced by ICH, and promotes the levels of VEGF and VEGFR-2 thereby improving neurological deficits and exaggerated brain water content induced by ICH.

\section{Materials and Methods}

Animal

All procedures of this study were approved by Institutional Animal Care \& Use Committee of Southwest Medical University, which were in compliance with the guidelines of the International Association for the Study of Pain. A total of fifty-three male Sprague-Dawley rats (200-250g) were used in this study and all animals were maintained under 12-hour light/dark cycle with free access to food and water in a 


\section{Cellular Physiology Cell Physiol Biochem 2017;41:145-153

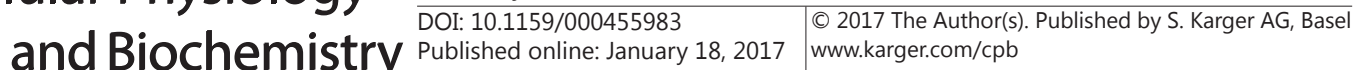

Wang et al.: Activation of mTOR by Intracerebral Hemorrhage

temperature- and humidity- controlled room. At the end of each experiment, overdose isoflurane was given to sacrifice the animals.

\section{Induction of ICH}

The rats were anesthetized by inhalation of isoflurane oxygen mixture $2-5 \%$ isoflurane in $100 \%$ oxygen), then immobilized in a stereotaxic apparatus (David Kopf, USA). After making a midline incision, the skull was exposed and one burr hole was drilled. Bacterial collagenase (type VII; Sigma Co.) was injected into the right basal ganglia (coordinates, $0.2 \mathrm{~mm}$ anterior, $6 \mathrm{~mm}$ ventral, and $3 \mathrm{~mm}$ lateral to the bregma). Collagenase [0.5 U in $2 \mu \mathrm{l}$ artificial cerebrospinal fluid (aCSF)] was infused into the brain over a period of 5 min via a Harvard pump before the injection needle was removed and the burr hole was covered by dental zinc cement. The same volume of aCSF was infused into the right basal ganglia as control.

In order to chronically deliver drugs into the brain tissues via intracerebroventricular (ICV) infusion, animals were cannulated with an L-shaped stainless steel cannula aimed the right lateral ventricle (coordinates: $3.7 \mathrm{~mm}$ posterior to the bregma, $4.1 \mathrm{~mm}$ lateral to the midline, and $3.5 \mathrm{~mm}$ under the dura). The guide cannula was fixed to the skull using dental zinc cement and jewelers' screw. The cannula was connected to an osmotic minipump (Alzet pump brain infusion kit, DURECT Inc., Cupertino, CA) with polycarbonate tubing. The pumps were placed subcutaneously between the scapulae, and loaded with aCSF as control and rapamycin, an inhibitor of mTOR. Rapamycin and aCSF were delivered at $0.25 \mu \mathrm{l}$ per hour (Alzet Model 1003D, a total of $100 \mu \mathrm{g}$ of rapamycin was given for 3 days). The dose and time point selected for rapamycin were based on results obtained from the prior reports [16-18]. This intervention allowed animals to receive continuous ICV infusion via the minipumps before brain tissues were removed.

Accordingly, the rats were divided into four groups. Group 1: control + aCSF $(n=10)$; group 2: control + rapamycin $(n=15)$; group 3: $\mathrm{ICH}+\operatorname{aCSF}(n=12)$ and group $4: \mathrm{ICH}+$ rapamycin $(\mathrm{n}=16)$. After completion of these treatments, the animals were anesthetized and sacrificed. The brains were removed to confirm the placement of the cannula and the right side of brain regions were dissected under an anatomical microscope and tissues were taken for the process.

\section{Western Blot Analysis}

The brain tissues were homogenized and centrifuged. A total protein was then extracted by homogenizing sample in ice-cold immunoprecipitation assay buffer with protease inhibitor cocktail kit. The lysates were centrifuged and the supernatants were collected for measurements of protein concentrations using a bicinchoninic acid assay reagent kit. After being denatured by heating at $95^{\circ} \mathrm{C}$ in an SDS sample buffer, the supernatant samples were loaded onto gels and then electrically transferred to a polyvinylidene fluoride membrane. The protein expression of mTOR, S6K1, 4E-BP1 and their respective phosphorylated forms, i.e. p-mTOR, p-S6K1, p-4E-BP1 as well as VEGFR-2 and Caspase-3 were determined using a standard Western blot analysis. In brief, membranes were incubated with rabbit anti-p-mTOR/p-S6K1/p-4E-BP1 antibodies; rabbit anti-mTOR/S6K1/4E-BP1 antibodies; and rabbit anti-VEGFR-2 and anti-Caspase-3 (1:500, obtained from Abcam Co.). After being fully washed, the membrane was incubated with horseradish peroxidaselinked anti-rabbit secondary antibody (1:250) and visualized for immunoreactivity. The membrane was also processed to detect $\beta$-actin for equal loading. The bands recognized by the primary antibody were visualized by exposure of the membrane onto an X-ray film. The film was then scanned and the optical densities of protein bands were analyzed using the Scion image software, and values for densities of immunoreactive bands $/ \beta$-actin from the same lane were determined. Each of the values was then normalized to a control sample.

\section{ELISA Measurements}

The levels of PICs were determined using an ELISA assay kit (Abcam Co.) according to the provided description and modification. Briefly, polystyrene 96-well microtiter immunoplates were coated with affinity-purified rabbit anti-IL-1 $\beta$, anti-IL-6 and anti-TNF- $\alpha$ antibodies, respectively. Parallel wells were coated with purified rabbit IgG for evaluation of nonspecific signal. After overnight incubation at room temperature and 2 hours of incubation with the coating buffer containing $50 \mathrm{mM}$ carbonate in $2 \%$ BSA, plates were washed with $50 \mathrm{mM}$ Tris- $\mathrm{HCl}$. The diluted samples and respective PIC standard solutions were distributed in each plate and left at room temperature overnight. The plates were washed and incubated with anti- IL-1 $\beta$, anti-IL- 6 and anti-TNF- $\alpha$ galactosidase. Then, the plates were washed and incubated with 


\section{Cellular Physiology Cell Physiol Biochem 2017;41:145-153

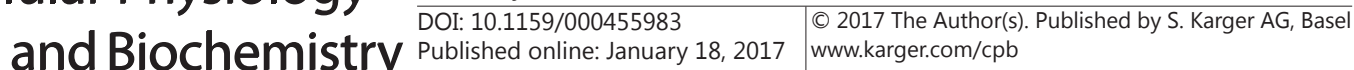 \\ Wang et al.: Activation of mTOR by Intracerebral Hemorrhage}

substrate solution. After an incubation of 2 hours at $37^{\circ} \mathrm{C}$, the optical density was measured using an ELISA reader. This method was also employed to examine the levels of VEGF according to the provided description and modification (Promega Corp. Madison, WI).

\section{Neurological Function and Brain Edema}

The modified method of Neurological Severity Score (mNSS) was used to examine neurological function 3 days after treatment. mNSS was generally used to assess a combination of motor, sensory, and balance functions $[9,19]$. Neurological function was graded on a scale of 0-18 (normal score, 0; maximal deficit score, 18). The rats with an abnormal mNSS (score $>0$ ) before ICH were excluded from the experiment.

Brain water content (brain edema) was determined 3 days after ICH. The brain slices (2 mm) were cut. The whole brain water content was calculated from all slices $[9,19]$. The brain slices were weighed to obtain the wet weight immediately and dried in an oven at $100^{\circ} \mathrm{C}$ for 24 hours to obtain the dry weight. The cerebellum was used as the internal control. The water content was expressed as the formula: [(wet weight) - (dry weight)] / (wet weight) $\times 100 \%$.

\section{Statistical Analysis}

A two-way repeated-measures analysis of variance was used to analyze all the data in this report as the data were obtained from two groups (control rats and ICH rats) with two different interventions (aCSF and rapamycin). Values were presented as means \pm standard error of mean. Differences were considered significant at $P<0.05$. All statistical analyses were performed using SPSS for Windows version 15.0 (SPSS, USA).

\section{Results}

\section{Expression of mTOR Pathway}

Figure 1 demonstrates the protein expression of p-mTOR, p-S6K1 and p-4E-BP1 as well as total protein levels of mTOR, S6K1 and 4E-BP1 in control rats infused with aCSF and ICH rats infused with aCSF three days after induction of ICH. ICH significantly increased the protein levels of p-mTOR and mTOR-mediated p-S6K1 and p-4E-BP1 in the brain tissues as compared with control rats infused with aCSF $(P<0.05 v$. control rats + aCSF; $n=6-12$ in each group). However, total protein levels of mTOR, S6K1 and 4E-BP1 were not significantly altered in ICH rats infused with aCSF $(P>0.05$, control rats + aCSF $v s$. ICH rats + aCSF $)$.

Effects of Blocking mTOR on the Levels of PICs

Figure 2 shows that IL-1 $\beta$, IL- 6 and TNF- $\alpha$ were significantly elevated in the brain tissues infused with aCSF after induction of ICH $(\mathrm{n}=12, P<0.05 \mathrm{vs}$. control rats + aCSF, $\mathrm{n}=$ 10 ) as compared with control rats infused with aCSF. Inhibition of mTOR by ICV infusion of rapamycin significantly decreased the amplification of IL-1 $\beta$, IL- 6 and TNF- $\alpha$ in ICH rats $(P<$ 0.05 , ICH rats with rapamycin $v s$. ICH rats with aCSF, $n=16$ with rapamycin and $n=12$ with aCSF). Infusion of rapamycin tended to attenuate expression of TNF- $\alpha$ in control rats infused with aCSF $(n=15)$, but no significant differences were observed $(P=0.105$, control rats with rapamycin $v s$. control rats with aCSF).

\section{Effects of Blocking mTOR on VEGF and VEGFR-2, and Caspase-3}

Figure $3 \mathrm{~A}$ illustrates that induction of ICH significantly increased the levels of VEGF in the brain tissues of rats infused with aCSF compared with control rats infused with aCSF $(P<$ 0.05 , ICH rats + aCSF vs. control rats + aCSF. $n=10$ in control + aCSF and $n=12$ in ICH group $+\mathrm{aCSF}$ ). Figure 3B further shows that ICH amplified the protein expression of VEGFR-2 and Caspase- 3 in the brain tissues of rats infused with aCSF $(P<0.05$ vs. control rats + aCSF $n$ $=6-12$ in each group). As rapamycin was infused into the brain of ICH rats to block mTOR signal pathway, VEGF and VEGFR-2 expression were enhanced and Caspase-3 expression was attenuated. Moreover, Fig. 3A shows that rapamycin failed significantly alter expression of VEGF in control animals infused with aCSF per se. 
Fig. 1. Expression of mTOR and its downstream signal. Typical bands (top panels) and averaged data (bottom panel): Showing p-mTOR, p-S6K1 and p-4E-BP1 in the brain tissues of control rats infused with aCSF and ICH rats infused with aCSF. Also, total protein of mTOR, S6K1 and 4E-BP1 was shown. ICH failed to significantly increase the total protein levels of mTOR, S6K1 and 4E-BP1, but significantly elevated p-mTOR, p-S6K1 and p-4E-BP1. * $\mathrm{P}<0.05$ vs. respective protein in control rats with aCSF. $n$ $=6-12$ in each group.
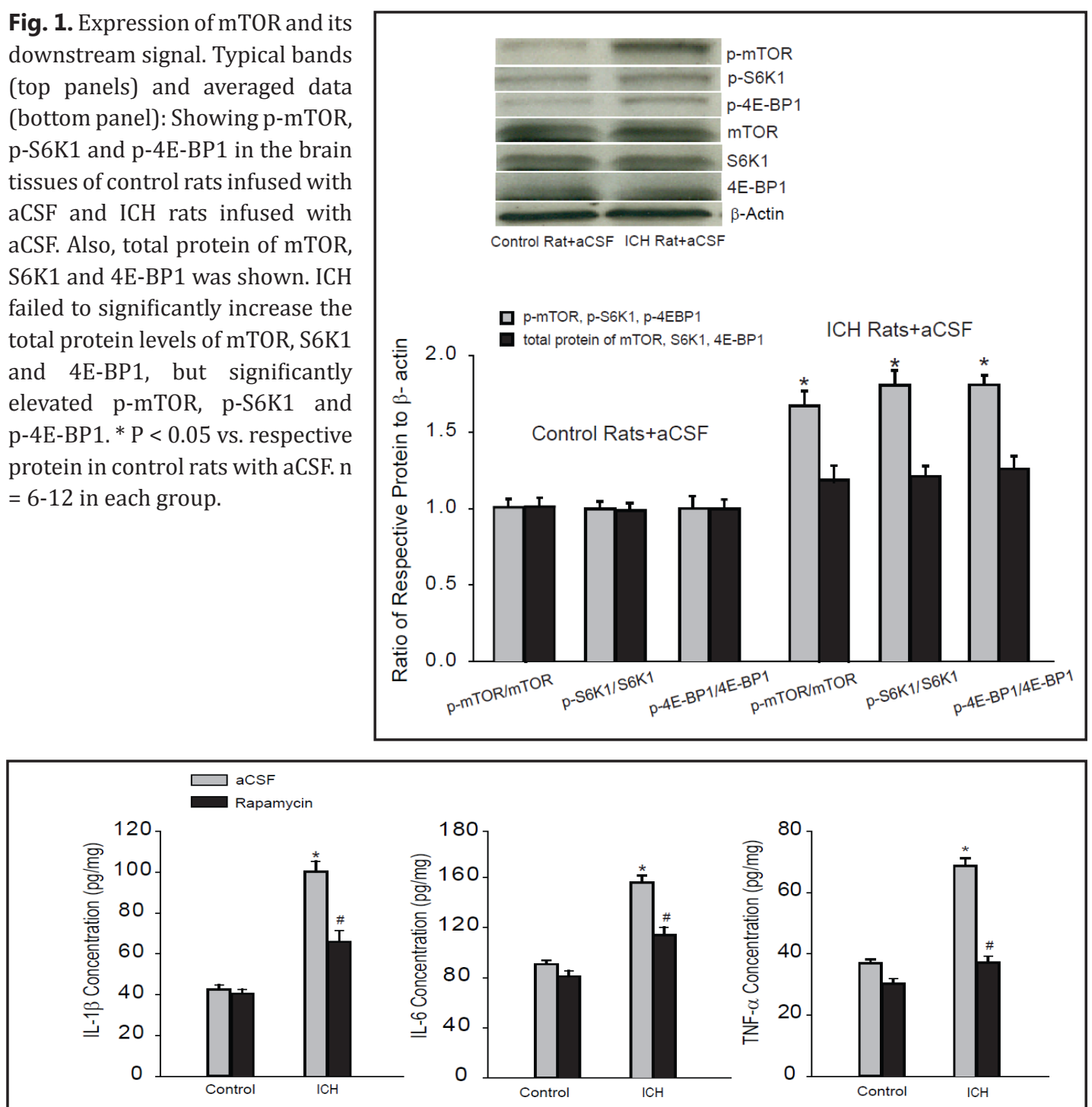

Fig. 2. Effects of blocking mTOR on PICs. The levels of IL-1 $1 \beta$, IL- 6 and TNF- $\alpha$ in the brain tissues. ICH amplified the levels of these PICs. Inhibition of mTOR signal with rapamycin decreased the augmented IL$1 \beta$, IL- 6 and TNF- $\alpha$ in brain tissues of ICH rats. ${ }^{*} \mathrm{P}<0.05$, ICH rats + aCSF $(n=12)$ vs. control rats + aCSF $(n$ $=10)$; and \# $\mathrm{P}<0.05$, rapamycin $(\mathrm{n}=16)$ vs. aCSF infusion in ICH rats $(\mathrm{n}=12)$. Note that rapamycin failed to significantly alter the levels of PICs in control rats infused with aCSF per se $(n=15)$.

\section{Neurological Function and Brain Edema}

Figure 3C\&D further demonstrate that ICH led to increases in the mNSS and water content $(P<0.05$, ICH rats with aCSF vs. control rats with aCSF; $\mathrm{n}=10$ in control + aCSF and $\mathrm{n}$ $=12$ in ICH group + aCSF). Also, the figures show that ICV infusion of rapamycin significantly attenuated the increased nNSS and amplified water content of brain tissues in ICH animals $(P<0.05$, rapamycin $v s$. aCSF infusion; $\mathrm{n}=16$ with rapamycin and $\mathrm{n}=12$ with aCSF $)$.

\section{Discussion}

A rat model of ICH has been employed to determine the mechanisms responsible for neurological damages. It is noted male rats were used in our current study, our data showed 
Fig. 3. Effects of blocking mTOR on VEGF, VEGFR-2, Caspase- 3 and nNSS and water content. (A): ICH increased the levels of VEGF in the brain tissues of rats infused with aCSF. ${ }^{*} \mathrm{P}<0.05$ vs. control rats + aCSF. $n=10$ in control $+\operatorname{aCSF}$ and $n=12$ in $\mathrm{ICH}+$ aCSF. Rapamycin amplified the levels of VEGF in ICH animals. \# $\mathrm{P}<0.05$, indicates aCSF vs. rapamycin in ICH rats. (B): ICH increased the protein expression of Caspase- 3 and VEGFR-2 in the brain tissues of rats infused with aCSF. ${ }^{*} \mathrm{P}$ $<0.05$ vs. respective control rats with aCSF. $n=6-12$ in each group. ICV infusion of rapamycin attenuated the enhanced protein expression of Caspase-3 induced by ICH and promoted VEGFR-2 expression. \# $\mathrm{P}<0.05$, indicates aCSF vs. rapamycin in ICH rats. (C) \& (D): ICH increased the mNSS and water content of brain tissues as compared with control animals infused with aCSF. ${ }^{*} \mathrm{P}<0.05$, ICH rats $+\operatorname{aCSF}(\mathrm{n}=12)$ vs. control rats $+\operatorname{aCSF}(\mathrm{n}=10)$. Rapamycin attenuated the increased nNSS and water content in ICH rats $(n=16)$. \# $P<0.05$ vs. ICH rats with aCSF infusion $(\mathrm{n}=12)$.

that expression of p-mTOR and its downstream pathways, namely p-S6K1 and p-4E-BP1 is upregulated by ICH. We further examined the effects of ICV infusion of rapamycin on a number of critical molecular mediators involved in the pathophysiological process of ICH including PICs, VEGF, VEGFR-2 and Caspase-3. We observed that rapamycin attenuates amplification of IL-1 $\beta$, IL- 6 and TNF- $\alpha$, and Caspase- 3 induced by ICH. In contrast, rapamycin enhances VEGF levels and VEGFR-2 expression in ICH animals infused with aCSF. Consistent with these results, our data further showed that damaged neurological scores and increased water content caused by ICH are significantly improved by rapamycin. Overall, we suggest that inhibition of mTOR signal pathway is likely to play a beneficial role in improving neurological deficits induced by ICH via PICs and VEGF mechanisms.

There are two distinct mTOR forms of protein complexes, mTORC1 and mTORC2. In general, mTORC1 is composed of raptor, MLST8 and mTOR, and is known to gate translation of most proteins by phosphorylation of specific downstream effectors including, p70 ribosomal S6 protein kinase (p70 S6Ks) and 4E-BPs [10]. mTOR, S6K1 and 4E-BP1 are expressed in the mammalian central nervous system [20-22]. The mTORC1 plays a critical role in the regulation of protein synthesis and growth and further the compelling evidence supports the notion that mTOR plays an important role in the modulation of long-term neuronal plasticity [20,23]. Specifically, data of our current study indicate the contribution of mTOR and its downstream effectors to the neuronal injuries in the process of ICH.

Neuroinflammation is involved in the pathophysiological process after ICH. The infiltration of specific immunocyte subtypes with phagocytic function is necessary to be into the ICH brain to clear degenerative and necrotic neurons and astrocytes post ICH $[24,25]$. In contrast, excessive inflammatory responses lead to detrimental effects contributing to 


\section{Cellular Physiology Cell Physiol Biochem 2017;41:145-153 \begin{tabular}{ll|l} 
DOI: 10.1159/000455983 & $\begin{array}{l}\text { O } 2017 \text { The Author(s). Published by S. Karger AG, Basel } \\
\text { www.karger.com/cpb }\end{array}$ \\
\hline Publisned online: January 18, 2017 Biochemistry
\end{tabular} \\ Wang et al:: Activation of mTOR by Intracerebral Hemorrhage}

secondary brain injuries post ICH [24, 25]. Among infiltrated immunocytes, neutrophils are the earliest immunocyte subtype to infiltrate into the brain after ICH and they appear within 4 to 5 hours and peak at day 3 in animal models of ICH [26-28]. Resident microglia cells in the brain are the first non-neuronal cells to respond to ICH induced damages. They become activated within 1 hour after ICH and peak at 3-7 days [24, 26]. Like neutrophils, microglia cells can clear up the hematoma by engulfing degenerative and necrotic cells and they can also secret pro-inflammatory cytokines including TNF $\alpha$ [27]. IL-1 $\beta$ and IL-6 recruit other immunocytes such as neutrophils and lymphocytes, which further aggravate brain injuries $[26,27]$. Evidence shows that inhibition of microglia cells could benefit the prognosis of ICH [28].

Inflammatory networks contributing to brain damage after ICH involve not only inflammatory cells, but also inflammation related cytokines [24, 28, 29]. Prior studies have indicated that increases in IL-1 $\beta$, IL- 6 and TNF- $\alpha$ induced by ICH exacerbate edema and brain damage by recruiting neutrophils and lymphocytes infiltrating into brain to destroy the integrity of blood brain barrier and other brain tissues; whereas anti-inflammatory cytokine IL-10 has beneficial effects against the brain damages following ICH [24-26, 29, $30]$. It was found that IL-1 $\beta$, IL- 6 and TNF- $\alpha$ decreased, while IL-10 elevated significantly in the peri-hematomal tissues of stem cell transplanted rats [31]. These results provided further evidence that stem cell grafts are able to not only suppress intracerebral infiltrations of inflammatory cells, but also effectively regulate the production and the secretion of inflammation-related cytokines in the peri-hematomal regions of ICH injured brain [31]. Consistently, data of our current study observed that neurological deficits and water content of brain tissues are improved in ICH animals after inhibition of mTOR and this links to attenuation of IL-1 $\beta$, IL- 6 and TNF- $\alpha$.

VEGF is an important signaling protein playing a role in regulating the neovascularization as a downstream product of HIF- $1 \alpha$, which contributes to VEGF formation due to ischemic or hypoxic conditions [11]. Also, VEGF and its subtype receptor VEGFR-2 play an important role in pathologic angiogenesis [32, 33]. Amplified HIF-1 $\alpha$, VEGF and VEGFR-2 have been found in the brain tissues after induction of ICH and promotion of VEGF and VEGFR-2 by stabilization of HIF- $1 \alpha$ can improve neurological deficits and increased brain water content induced by ICH [18]. Nevertheless, results of our current study provide evidence that blocking of mTOR signal plays a beneficial role in modulating brain injuries observed in ICH via VEGF mechanisms.

Caspases, a family of thiol proteases, play an important regulating the apoptotic cascade, and induction of ICH activates neuronal caspases. In the death-signaling cascade, it is crucial that pro-caspase- 3 is processed to its active form Caspase-3, and it has been reported that Caspase- 3 is a principal target that is engaged in the reactive oxygen species-mediated apoptosis in human endothelial cells [34]. Thus, in the current study, we also determined the levels of Caspase-3 in brain tissues as an indicator of cellular apoptosis and we found that ICH increases Caspase-3 in ICH animals infused with aCSF. Moreover, inhibition of mTOR decreases the amplified expression of Caspase-3 evoked by ICH.

\section{Conclusions}

Activation mTOR signal is involved in pathophysiological process during ICH and blocking mTOR pathway plays a role in improving deteriorated neuronal tissues via PIC and VEGF mechanisms. Specifically, results of this study have pharmacological implications to target mTOR signal pathway for neuronal dysfunction and vulnerability related to ICH.

\section{Disclosure Statement}

None.

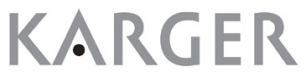




\section{Cellular Physiology Cell Physiol Biochem 2017;41:145-153

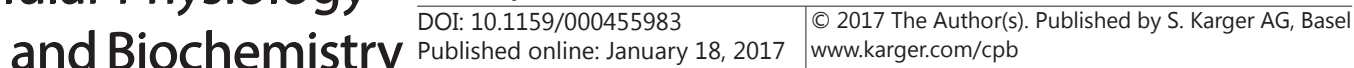

Wang et al.: Activation of mTOR by Intracerebral Hemorrhage

\section{References}

1 van Asch CJJ, Luitse MJA, Rinkel GJE, van der Tweel I, Algra A, Klijn CJM: Incidence, case fatality, and functional outcome of intracerebral haemorrhage over time, according to age, sex, and ethnic origin: a systematic review and meta-analysis. Lancet Neurol 2010;9:167-176.

2 Hoff J, Xi G: Brain edema from intracerebral hemorrhage; in (Kuroiwa T, Baethmann A, Czernicki Z, Hoff JT, Ito U, Katayama Y, Marmarou A, Mendelow BAD, and Reulen HJ, eds) Brain Edema XII. Springer Vienna, 2003, vol. 86, pp. 11-15.

-3 Rincon F, Mayer SA: Novel therapies for intracerebral hemorrhage. Cur Opin Crit Care 2004;10:94-100.

$\checkmark 4$ Hill MD: Stroke and diabetes mellitus. Handb Clin Neurol 2014;126:167-174.

-5 Fan X, Jiang Y, Yu Z, Yuan J, Sun X, Xiang S, Lo EH, Wang X: Combination approaches to attenuate hemorrhagic transformation after tPA thrombolytic therapy in patients with poststroke hyperglycemia/ diabetes. Adv Pharmacol 2014;71:391-410.

6 Tapia-Perez JH, Gehring S, Zilke R, Schneider T: Effect of increased glucose levels on short-term outcome in hypertensive spontaneous intracerebral hemorrhage. Clin Neurol Neurosurg 2014;118:37-43.

7 Zhang G, Wu F, Xu Y, Feng J, Cai Z, Xu B, Zhou X, He M: Prestroke glycemic status is associated with the functional outcome in spontaneous intracerebral hemorrhage. Neurol Sci 2015;36:927-934.

8 Ding G, Yan T, Chen J, Chopp M, Li L, Li Q, Cui C, Ning R, Jiang Q: Persistent cerebrovascular damage after stroke in type two diabetic rats measured by magnetic resonance imaging. Stroke 2015;46:507-512.

-9 Xu S, Wu Q, Guo G, Ding X: The protective effects of urocortin1 against intracerebral hemorrhage by activating JNK1/2 and p38 phosphorylation and further increasing VEGF via corticotropin-releasing factor receptor 2. Neurosci Lett 2015;589:31-36.

10 Hay N, Sonenberg N: Upstream and downstream of mTOR. Genes Dev 2004;18:1926-1945.

11 Kim Y-W, Byzova TV: Oxidative stress in angiogenesis and vascular disease. Blood 2014;123:625-631.

12 Hua-Jun Zhou, Tao Tang, Han-Jin Cui, A-Li Yang, Jie-Kun Luo, Yuan Lin, Qi-Dong Yang, Xing-Qun Li: Thrombin-triggered angiogenesis in rat brains following experimental intracerebral hemorrhage. J Neurosurg 2012;117:920-928.

13 Peng Z-R, Yang AL, Yang Q-D: The effect of hyperbaric oxygen on intracephalic angiogenesis in rats with intracerebral hemorrhage. J Neurol Sci 2014;342:114-123.

14 Holmes K, Roberts OL, Thomas AM, Cross MJ: Vascular endothelial growth factor receptor-2: Structure, function, intracellular signalling and therapeutic inhibition. Cell Sig 2007;19:2003-2012.

15 Stuttfeld E, Ballmer-Hofer K: Structure and function of VEGF receptors. IUBMB Life 2009;61:915-922.

16 Chauhan A, Sharma U, Jagannathan NR, Reeta KH, Gupta YK: Rapamycin protects against middle cerebral artery occlusion induced focal cerebral ischemia in rats. Behav Brain Res 2011;225:603-609.

17 Lu Q, Gao L, Huang L, Ruan L, Yang J, Huang W, Li Z, Zhang Y, Jin K, Zhuge Q: Inhibition of mammalian target of rapamycin improves neurobehavioral deficit and modulates immune response after intracerebral hemorrhage in rat. J Neuroinflammation 2014;11:44.

18 Yu Z, Tang L, Chen L, Li J, Wu W, Hu C: Role for HIF-1alpha and Downstream Pathways in Regulating Neuronal Injury after Intracerebral Hemorrhage in Diabetes. Cell Physiol Biochem 2015;37:67-76.

19 Zhang Y, Yi B, Ma J, Zhang L, Zhang H, Yang Y, Dai Y: Quercetin promotes neuronal and behavioral recovery by suppressing inflammatory response and apoptosis in a rat model of intracerebral hemorrhage. Neurochem Res 2015;40:195-203.

-20 Costa-Mattioli M, Sossin WS, Klann E, Sonenberg N: Translational control of long-lasting synaptic plasticity and memory. Neuron 2009;61:10-26.

21 Zhang C, Yuan XR, Li HY, Zhao ZJ, Liao YW, Wang XY, Su J, Sang SS, Liu Q: Anti-cancer effect of metabotropic glutamate receptor 1 inhibition in human glioma U87 cells: involvement of PI3K/Akt/mTOR pathway. Cell Physiol Biochem 2015;35:419-432.

22 Cao L, Tian Y, Jiang Y, Zhang GJ, Lei H, Di ZL: Down-regulation of Homer1b/c protects against chemically induced seizures through inhibition of mTOR signaling. Cell Physiol Biochem 2015;35:1633-1642.

23 Banko JL, Poulin F, Hou L, DeMaria CT, Sonenberg N, Klann E: The translation repressor 4E-BP2 is critical for eIF4F complex formation, synaptic plasticity, and memory in the hippocampus. J Neurosci 2005;25:9581-9590.

24 Aronowski J, Zhao X: Molecular pathophysiology of cerebral hemorrhage: secondary brain injury. Stroke 2011;42:1781-1786. 


\section{Cellular Physiology Cell Physiol Biochem 2017;41:145-153

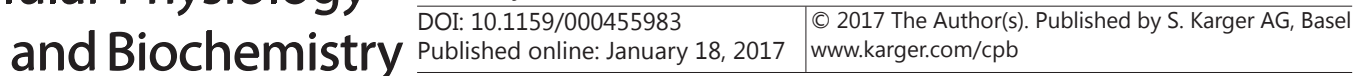 \\ Wang et al.: Activation of mTOR by Intracerebral Hemorrhage}

25 Keep RF, Hua Y, Xi G: Intracerebral haemorrhage: mechanisms of injury and therapeutic targets. Lancet Neurol 2012;11:720-731.

-26 Lee ST, Chu K, Jung KH, Kim SJ, Kim DH, Kang KM, Hong NH, Kim JH, Ban JJ, Park HK, Kim SU, Park CG, Lee SK, Kim M, Roh JK: Anti-inflammatory mechanism of intravascular neural stem cell transplantation in haemorrhagic stroke. Brain 2008;131:616-629.

-27 Shichita T, Ago T, Kamouchi M, Kitazono T, Yoshimura A, Ooboshi H: Novel therapeutic strategies targeting innate immune responses and early inflammation after stroke. J Neurochem 2012;123:29-38.

-28 Wang J: Preclinical and clinical research on inflammation after intracerebral hemorrhage. Prog Neurobiol 2010;92:463-477.

29 Hwang BY, Appelboom G, Ayer A, Kellner CP, Kotchetkov IS, Gigante PR, Haque R, Kellner M, Connolly ES: Advances in neuroprotective strategies: potential therapies for intracerebral hemorrhage. Cerebrovasc Dis 2011;31:211-222.

-30 Ziai WC: Hematology and inflammatory signaling of intracerebral hemorrhage. Stroke 2013;44:S74-78.

-31 Qin J, Ma X, Qi H, Song B, Wang Y, Wen X, Wang QM, Sun S, Li Y, Zhang R, Liu X, Hou H, Gong G, Xu Y: Transplantation of Induced Pluripotent Stem Cells Alleviates Cerebral Inflammation and Neural Damage in Hemorrhagic Stroke. PLoS One 2015;10:e0129881.

-32 Finger RP, Guymer RH, Gillies MC, Keeffe JE: The Impact of Anti-Vascular Endothelial Growth Factor Treatment on Quality of Life in Neovascular Age-Related Macular Degeneration. Ophthalmology 2014;121:1246-1251.

33 Mintz-Hittner HA, Kennedy KA, Chuang AZ: Efficacy of intravitreal bevacizumab for stage 3+ retinopathy of prematurity. N Engl J Med 2011;364:603-615.

-34 Ho FM, Liu SH, Liau CS, Huang PJ, Lin-Shiau SY: High glucose-induced apoptosis in human endothelial cells is mediated by sequential activations of c-Jun NH(2)-terminal kinase and caspase-3. Circulation 2000;101:2618-2624. 